\title{
Data Transmission Using Gyrotron Radiation as a Carrier
}

\author{
A. Fokin, A. Sedov, A. Tsvetkov \\ ${ }^{1}$ Institute of Applied Physics of the Russian Academy of Sciences, Nizhny Novgorod, Russia, ap.fokin@mail.ru
}

The idea of advancement of data transmission systems to the sub-THz region is actively discussed in the scientific and engineering community $[1,2]$. The available frequencies are limited by the absorption in the atmosphere, so the range of 200-300 GHz seems very promising. But for this frequency range the complication in the implementation of communication systems is the lack of medium-power (up to $1 \mathrm{~kW}$ ) sources with wide tuning capabilities. In this paper we present the first experiments using the sub-THz gyrotron as the radiation source for data transmission.

\section{Gyrotron and control system}

The experiments were carried out on the $263 \mathrm{GHz}$ gyrotron for spectroscopy and media diagnostics with output power up to $1 \mathrm{~kW}$ [3]. The control of output power and frequency was performed by variation of anode voltage of the triode-type magnetron-injection gun. Such system was already used for frequency stabilization of the gyrotron [4] and is capable of modulating parameters of gyrotron radiation with modulation frequency up to $1 \mathrm{MHz}$, defined by the time constant of the circuit. The anode voltage control unit is capable of variation of anode voltage directly proportional to the input signal with amplitude of $0-10 \mathrm{~V}$.

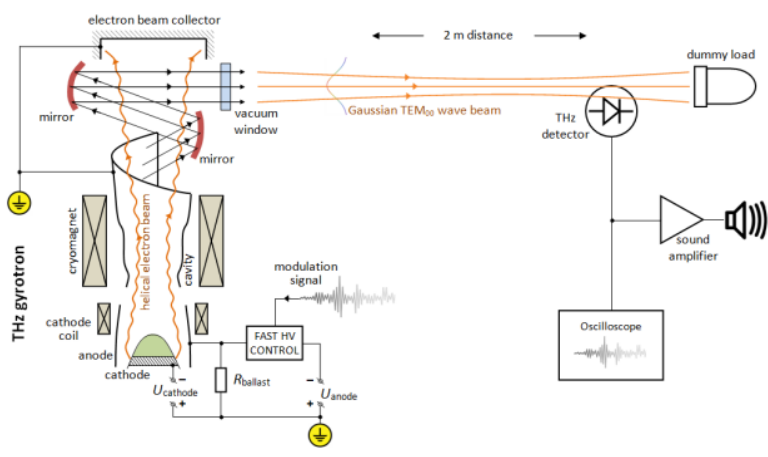

Fig. 1. The diagram of the experimental setup

\section{Experimental test}

The diagram of the experimental setup is presented in the fig. 1. In the first experiments the simple amplitude modulation of the gyrotron radiation was used. Detection of the modulated sub- $\mathrm{THz}$ radiation at the distance of $2 \mathrm{~m}$ was performed by an ultrafast $\mathrm{THz}$ detector produced by TeraSense company. We used two types of input data signals: an analog sound signal, taken from the loudspeaker exit of the car stereo, and the pseudo-random bit sequence, formed by the arbitrary waveform generator Agilent 33521B.

In the experiment we demonstrated the possibility of exact transmission of audio signal, using the gyrotron radiation as a carrier, which is justified by the analysis of the resulting oscilloscope traces of the input and resulting signal and by direct playback of the received signal. For the digital data transmission, we demonstrated the maximum rate of about 1.5 Mbit/s. The simple estimates of the system show the possibility of the increase of transmission rates up to 1 Gbit/s with minor upgrade of the control unit.

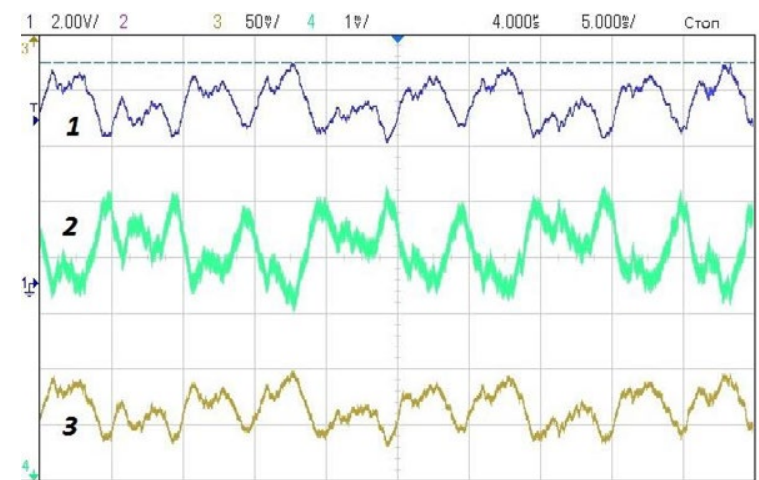

Fig. 2. Sound transmission: input analog sound signal (1) signal from detector (2) and anode voltage (3)

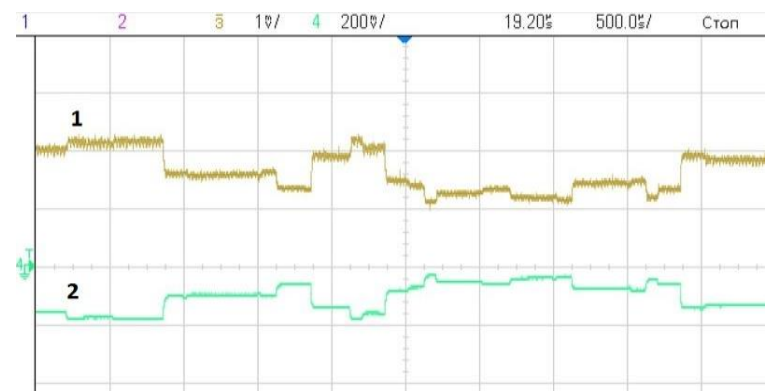

Fig. 3. Pseudo-random bit sequence: signal of the $\mathrm{THz}$ detector (1) and input sequence (2)

\section{Conclusion}

The possibility of data transmission using the modulation of the gyrotron radiation was demonstrated. The precise transmission of audio signal and 1.5 $\mathrm{Mbit} / \mathrm{s}$ data rate for digital signal were obtained.

The work was partially supported by the Russian Federation President Grant No. MK-3452.2017.8

\section{References}

1. T. Schneider et al., Link budget analysis for Terahertz fixed wireless links // IEEE Trans. on Terahertz Science and Technology, 2, 250 (2012)

2. T. Kürner and S. Priebe, Towards THz communications-status in research, standardization and regulation, J. Infrared, Millimeter, and Terahertz Waves, 35, 53-62 (2014)

3. M. Glyavin et al. Experimental tests of $263 \mathrm{GHz}$ gyrotron for spectroscopy applications and diagnostic of various media // Review of Scientific Instruments, 86, No. 5, 054705 (2015)

4. A. Fokin et al. High-power sub-terahertz source with a record frequency stability at up to $1 \mathrm{~Hz} / /$ Scientific Reports, 8, 4317 (2018) 